\title{
Research in clinical psychiatry: an interdisciplinary view from Germany
}

\author{
Molecular Psychiatry (2004) 9, 541. doi:10.1038/sj.mp. \\ 4001526
}

The editor asked in his January 2004 editorial $^{1}$ why psychiatry in America may have failed to integrate clinical and basic sciences, and questioned the reasons why the excellence in neuroscience research appears to be disconnected from clinical practice. This is, not surprisingly, similar to what has happened in Germany, but there seems to be reason for cautious optimism at the moment.

More than 30 years ago Gerd Huber merged the Weißenau clinics, a former typical state hospital, with a University department, although with low means as compared with other (typical) University departments. Nevertheless, other clinics followed, including our clinic in Günzburg, which became integrated as a department to the University of Ulm. The idea to place research directly into hospitals, the latter responsible for full regional psychiatric care, remained both attractive and difficult over the years: research remained supported at an excessively low level, whereas experience with a high number of severely diseased patients remained at a high level. Presently, the classical psychiatric University departments have taken over or are taking over full responsibility for psychiatric care within small specific regions, becoming in a way similar to state hospitals; however, with a reversed relationship regarding the burden of research vs clinical obligations for the staff. Such a department structure may prove preferable compared to the previous model, and may lead to the development of powerful and integrated psychiatric research centers. Nevertheless, the old model of state hospitals merged to the University department may still be useful provided there is better support for research.

My own experience of 24 years in this type of departmental structure has been that burden of care for severely diseased patients has unique advantages: severely diseased patients are most often treated in state clinics; patients can be seen and are treated often over months or even years as inpatients, some are seen over years or decades repeatedly as in- and outpatients or as clients of shelters, which receive medical support from the hospital. Such experience gives views different from that of short-term patients. However, this model was not satisfactory for research. Even though one can find scientific partners in frontier research groups in neurovirology, immunology or biochemistry and physiology, as I did, collaborating with many of them over decades, to establish and maintain such cooperative and integrative interdisciplinary work is challenging. Moreover, such long-term approaches are poorly supported if at all, but are truly necessary, in spite of insufficient funding, because psychiatric disorders develop slowly and a sufficient number of patients seen over the long term is difficult to collect. The longitudinal complexity of psychiatric disorders requires vast experience that is necessary to understand them at clinical and research levels.

I believe that even relatively modest budgets that are guaranteed over long-term periods could be extremely beneficial, particularly in the context of continuous integration and collaboration in emerging areas. This approach was utilized during the Eighth Psychoimmunology Expert Meeting-crosstalk with neurovirology, immunology and neurology (2003), which was perceived as truly successful. The participants in this type of interdisciplinary forums usually enjoy the integration of seemingly divergent fields, which would otherwise not interact in the same setting.

Why is interdisciplinary research so difficult? Several factors may be involved: to bring together different approaches is intrinsically difficult; biological-psychological pathways and interactions are extremely intricate; the underlying biochemistry is complex enough, but exponentially so when considering networks and interactions of neural and other cells, such as glia and immune cells. Additionally, redundancy in many biochemical pathways is very frequent and hard to tackle experimentally. Considerable amounts of active collaborative effort and openness for new ideas are required to address such complexity. Furthermore, in the clinical field, many basic requirements of modern scientific approaches are difficult or impossible to apply. The practical and ethical limitations of clinical approaches may be derided by basic scientists. However, the experienced clinician may have unique insights into features of disease that have not yet been elucidated by basic science. Basic scientists also have to deal with the limitations of interdisciplinary research when trying to participate in multidisciplinary efforts to increase knowledge of disease. The key issue for both clinicians and basic scientists is whether scientific progress can help patients. The gratification of interacting collaboratively is particularly important in interdisciplinary research but alone it will not suffice to improve integration of clinical and basic sciences. Increased funding for interdisciplinary cooperative work is of paramount importance for the advancement of psychiatry.

Karl Bechter ${ }^{1,2}$

${ }^{1}$ Department of Psychosomatics/Psychotherapy,

The Clinic for Psychiatry and Psychotherapy,

Bezirkskrankenhaus, Günzburg; ${ }^{2}$ Department Psychiatry II, University of Ulm, Ludwig-Heilmeyer-Str 2, Günzburg,

Germany

E-mail: Karl.Bechter@bkh-guenzburg.de

\section{Reference}

1 Licinio J. Mol Psychiatry 2004; 9: 1. 